\title{
The Use of ZigBee Wireless Communication Technology in Industrial Automation Control
}

\author{
ShuYu Ding $\mathbb{D}^{1}{ }^{1}$ JianLi Liu, ${ }^{2}$ and MingHong Yue ${ }^{3}$ \\ ${ }^{1}$ School of Network and Communication Engineering, Chengdu Technological University, Chengdu, 611730 Sichuan, China \\ ${ }^{2}$ School of Geophysics, Chengdu University of Technology, Chengdu, 610059 Sichuan, China \\ ${ }^{3}$ Technical Support Department, Heilongjiang Sub-Bureau of Northeast China Regional Air Traffic Management Bureau, CAAC, \\ Harbin, 150000 Heilongjiang, China
}

Correspondence should be addressed to ShuYu Ding; dsyu1@cdtu.edu.cn

Received 30 October 2021; Accepted 27 November 2021; Published 23 December 2021

Academic Editor: Haibin Lv

Copyright (c) 2021 ShuYu Ding et al. This is an open access article distributed under the Creative Commons Attribution License, which permits unrestricted use, distribution, and reproduction in any medium, provided the original work is properly cited.

With the accelerating process of world industrialization, countries pay more attention to industrial modernization. In order to solve the problems of traditional control technology and system with low accuracy, high energy consumption, and high delay in industrial automation control and the difficulty in adapting to the new industrial production process, the new industrial automation control system is discussed and studied. The industrial automation control is studied, and a new monitoring system is designed for automation control by using ZigBee wireless communication technology. The design of the system data communication scheme is completed by the in-depth study and summary of ZigBee wireless communication technology. The hardware structure and software structure of ZigBee wireless communication module are designed, and the wireless monitoring system using ZigBee is established. Taking the production workshop of a pharmaceutical factory as the monitoring object, the working stability and data transmission efficiency of the model are tested. The experiment shows that the ZigBee wireless transmission model has lower temperature and better stability than the wired transmission system in the working process. Compared with the traditional wired control system, the temperature of ZigBee wireless control system is lower when it works. The difference between the highest temperature and the lowest temperature is $0.1^{\circ} \mathrm{C}$. In the overall work flow, the system temperature is lower than that of the wired control. ZigBee wireless control has good stability. Subsequently, the method of manually setting obstacles is used to test the effectiveness of data transmission. The experiment found that in the absence of barrier, the maximum effective transmission distance was 40 meters, and the packet loss rate was very low, which could ensure the timeliness of data. Therefore, this system can be applied to industrial automation control. The research results can provide some reference for the design of new control systems in other industrial control fields. The improved system can also be applied to other industries. ZigBee is a short-range technology for wireless sensor networks, and it is a two-way communication technology with low power consumption, low data rate, and low cost. It can be applied to automatic control and remote control. This provides a platform for wireless networking and control of small and cheap devices. ZigBee will not compete with connection technologies such as Wi-Fi. Meanwhile, it will greatly stimulate the progress of the industrial market.

\section{Introduction}

As a big manufacturing country, China's industry occupies a large proportion in the economic system and has gradually become an important pillar industry of China's economic development. From the current development process of developed countries, industrialization is an indispensable process [1]. Therefore, China has always attached great importance to industry. With the emergence of various high-tech in the world, the times require China to transform from an industrial power to an industrial power. But there is still a big gap between China and developed countries in terms of industrial capacity and technology, so industrial automation has become an essential goal [2].

At present, there are two types of industrial automation technology: One is programmable logic controller (PLC). 
Zhao used PLC control technology in electronic automatic control. The PLC power automation control system was designed, and the system was simulated and tested [3]. The other is the distributed control system (DCS). Aparna (2020) used DCS control technology to design a nonlinear interactive multi-input, multioutput system battery [4]. With the advancement of technology and the improvement of computer performance, the automation control of industrial automation and wireless and wired communication gradually merge and achieve data synchronization. Fernandez et al. used time-sensitive networks to design the industrial application technology of media access control for wired communication [5]. Although the technical security of wired transmission is strong and the delay is low, the data has high timeliness and reliability [6]. However, in actual situations, the wiring method of wired transmission is easily restricted by the scene. Once the line is damaged, it may cause damage to the system and data [7]. Therefore, wireless communication technology has been gradually applied in industrial automatic control systems, and many kinds of sensing technologies have been derived. ZigBee is a new type of short-distance wireless technology [8]. Abdulkarem et al. [9] introduced a new technology for structural health monitoring by wireless sensor networks that offers advantages over traditional wired systems. The advantage of the system is to reduce the installation and maintenance costs of the structural health monitoring system. Open research issues in wireless sensor networks for structural health monitoring are discussed. Li and Liu [10] established a reasonable mathematical model. Additionally, aiming at the problem of poor coverage of existing algorithms, they proposed a monitoring area coverage optimization algorithm by the sensing probability around nodes. The experiments show that the algorithm has good performance, which can reduce the moving distance of nodes, improve the coverage range of the network, and extend the service time of the whole network. Because of this, the application of ZigBee technology in industrial automatic control is being studied.

Firstly, relevant documents are retrieved, and the theoretical knowledge of ZigBee technology is summarized. By copying the concept learning through the structure frame diagram of ZigBee technology, the three structures of ZigBee network are analyzed, and the address allocation rules of ZigBee network are expounded. The optional network structure of ZigBee technology is studied. Subsequently, using ZigBee, communication modules and wireless sensor models were designed. Combined with the requirements of industrial control technology, a model of the production workshop of the pharmaceutical factory was established. The data transmission efficiency and working state stability of ZigBee wireless transmission technology are analyzed. The stability of ZigBee wireless transmission technology is expressed by the temperature of the system. In this way, the performance stability of ZigBee industrial automation control system can be judged. The innovation of the method proposed lies in exploring the efficiency of wireless transmission by setting obstacles during wireless transmission and using temperature to demonstrate the stability of ZigBee wireless technology.

\section{Materials and Methods}

2.1. ZigBee Technology. ZigBee protocol communication equipment is divided into three types: coordinator, router, and terminal equipment. Generally, each complete ZigBee network consists of a coordinator and numerous routers and terminals. [11] The coordinator is generally by a full functional device (FFD) [12]. The function of coordinator is to initialize the network and form the ZigBee network. First, the coordinator scans the surrounding channel and selects the high energy value as the alternative channel. Then, the coordinator filters the channels with fewer nodes to establish the network and sets the corresponding network parameters. It can also be used as a router, mainly controlling the entire network, so stable energy supply is needed. Routers are generally set in the middle area of ZigBee network, responsible for the discovery and selection of data when passing, and the most suitable path for data transmission. The function of terminal equipment is relatively simple [13], which is generally at the end of the network and is responsible for data collection and transmission at the edge of the network. Terminal equipment can save energy by sleeping. The network structure of ZigBee is shown in Figure 1.

In Figure 2, there are generally three ZigBee network structures, a star structure, a mesh structure, and a tree structure.

The star structure [14] is to connect several terminal devices and nodes to the same coordinator. This device can be another complete system or a simple networked device. Since the star structure is multiple devices connected to the same coordinator, it is generally used in short-distance communication scenarios, such as various intelligent electrical and general control systems in the home. The star structure system management is very simple. When one device fails, it will not affect the normal operation of other devices. The disadvantage of the star structure system is that it is easy to cause too much burden on the coordinator and cause network congestion.

The mesh structure [15] is a directional path diagram that connects a coordinator and many nodes or devices, and the internal devices and nodes can communicate autonomously. Therefore, the mesh structure has the characteristics of high flexibility, is a structure that can support a variety of hop nodes, and is suitable for large-scale network structures. The disadvantage of the mesh structure system is that it requires a large amount of data and information to support operation, which increases energy consumption.

In the tree structure [16], the position of the coordinator is at the root, playing the same role as the root of the tree. We connect the remaining nodes as child nodes to form a multilayered one-to-many parent-child hierarchical relationship and transmit data according to the parent-child relationship. The tree structure is usually used for longdistance data transmission. Because the tree structure does not have the routing function, the data can only be transmitted layer by layer in the order of the assigned nodes, so the tree structure consumes less energy, but the transmission delay will be slightly higher. 


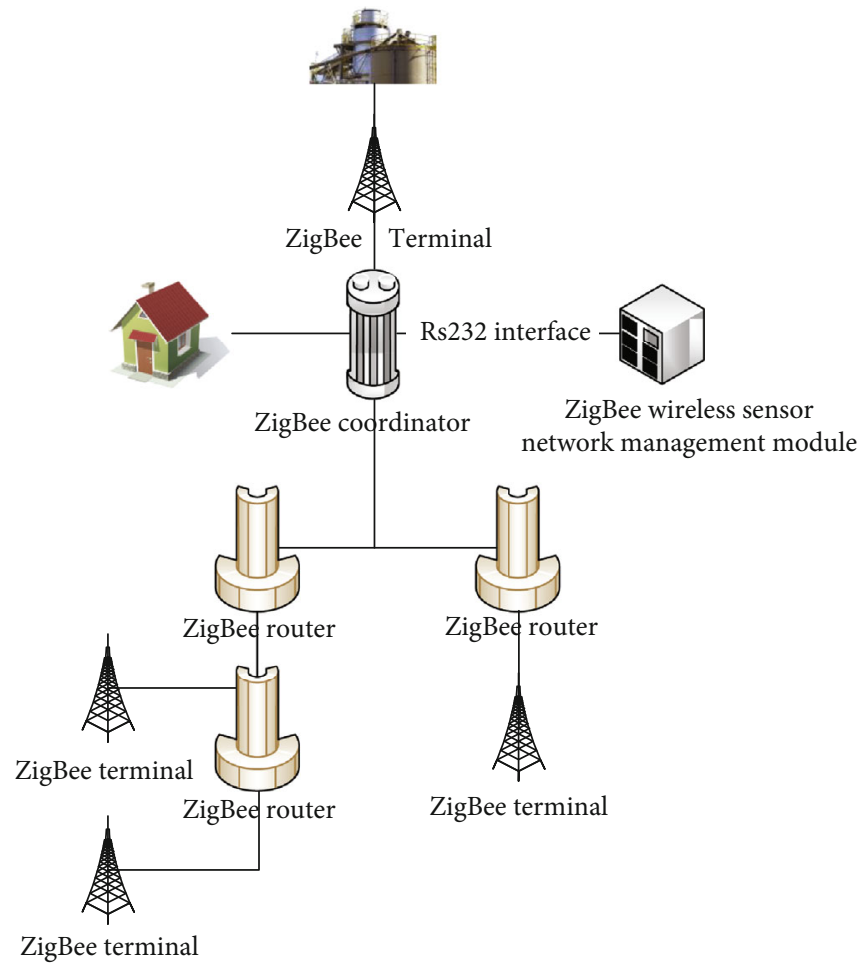

Figure 1: Basic structure diagram of ZigBee network.

There are generally two ZigBee address allocation rules. One is the 64-bit Institute of Electrical and Electronics Engineers (IEEE) extended address [17], and its coordinator network address is 0 . The other is the address of the parent node when the node is connected to the network through the distributed address assignment mechanism (DAAM) [18]. $C_{\text {skip }}(d)$ is a parameter in the DAAM allocation rule. It is the address offset of the child node, which means the interval between the coordinator and the node at depth $\mathrm{d}$, which is left for the node address at depth $d+1$ space. The address of the first child node that joins the parent node is parent node +1 , and the calculation of $C_{\text {skip }}(d)$ satisfies

$$
C_{\text {skip }}(d)=\left\{\begin{array}{l}
1+C_{m} *\left(L_{m}-d-1\right), R_{m}=1, \\
\frac{1+C_{m}-R_{m}-C_{m} * R_{m}{ }^{L_{m}-d-1}}{1-R_{m}}, \text { Other, }
\end{array}\right.
$$

where $C_{m}$ is the maximum number of child nodes that the parent node can load. $R_{m}$ is the maximum number of nodes that the child node can load. $L_{m}$ is maximum depth of ZigBee network. Refer to the DAAM allocation rules, assuming that the address of the parent node is $A_{p}$, then, the address of the $n$th access parent node is

$$
A_{n}=A_{p}+1+C_{\text {skip }}(d) *(n-1) .
$$

If the $n$th node is the end point, the address is

$$
A_{n}=A_{p}+1+C_{\text {skip }}(d) * R_{m}+n \text {. }
$$

ZigBee routing protocol [19] is the core technology of ZigBee. During data transmission, a node can transmit to its parent node or child node along the tree structure according to the connection between the destination address and the current address. Assuming that the current node address is $A$, the network depth is $d$, and the end point address is $D$, then, the method for judging whether the end point is a child node is as shown in

$$
A<D<A+C_{\text {skip }}(d) .
$$

If the end node address satisfies Equation (4), it means that the end node is the child node of the current node, and the next URL N can be calculated, as in Equation (5):

$$
N=A+1+\left[\frac{D-(A+1)}{C_{\text {skip }}(d)}\right] \cdot C_{\text {skip }}(d),
$$

where $C_{\text {skip }}(d)$ is a parameter in DAAM allocation rules, which is the address offset of the child node. It means the interval between coordinator and node in depth $d$. It is the space left for the node address with depth $d+1$, and the first child node to join the parent node is the parent node +1 . The address of the node is $A$, the network depth is $d$, and the endpoint address is $D$. Whenever the source node of the router transmits data to the destination node, the router of the source node does not store the information of the destination node and enters the route discovery stage. The source node writes own information and routing requirements into the system and transmits it to the entire network. When the intermediate node accepts it for the first time, it 


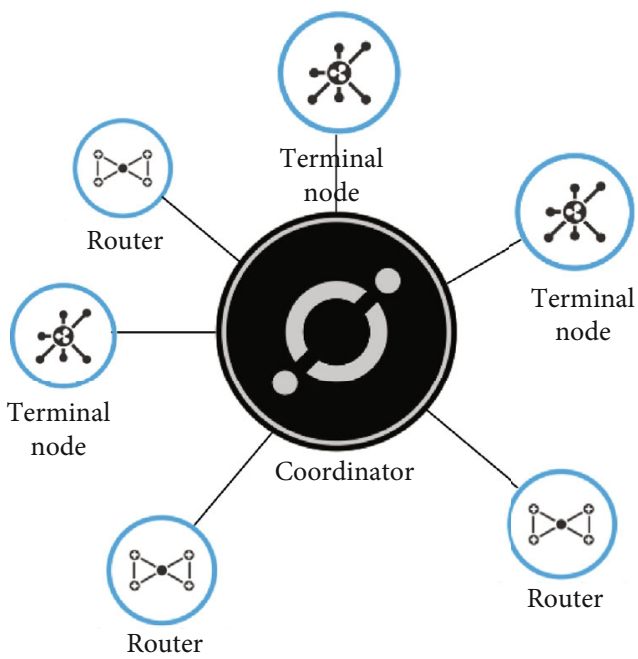

(a)

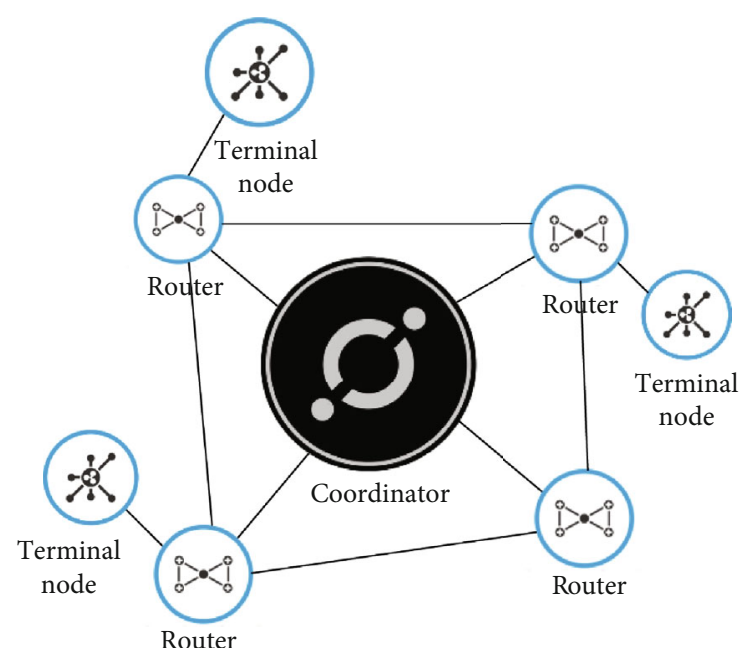

(b)

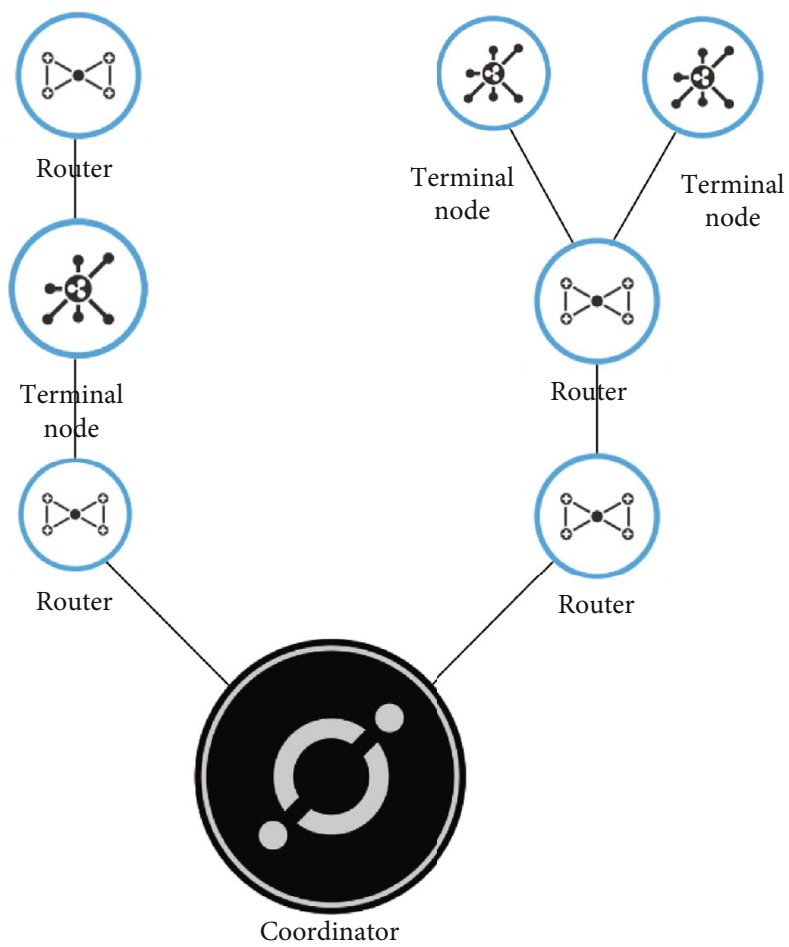

(c)

Figure 2: Three technologies of ZigBee wireless communication network ((a) is a star structure, (b) is a mesh structure, and (c) is a tree structure).

will retain the information in the packet, combine it with the address information of the previous node to form a reverse routing path from this node to the source node, and then transmit it to the broadcast. When the end node receives the information for the first time, it returns the packet to reply. If the node is not receiving it for the first time, it will be discarded as spam. Figure 3 shows the three transmission modes of route discovery, reverse routing, and forward routing.

2.2. Design and Establishment of ZigBee Wireless Sensor System. In the design of the ZigBee system structure, the cur- rent wired communication network of a pharmaceutical factory is improved, and the use of a wireless system can effectively solve the problem of complex wiring. A pharmaceutical factory production workshop was used as a model, and a wireless sensor was designed to monitor the status of the workshop. Figure 4 is an overall structural diagram. First, the sensor collects the data transmitted by the field networked devices and processes the information transmitted by the router. The router is responsible for network data transmission to ensure stable and correct data transmission between various devices. The coordinator is responsible for collecting data and sending it to the host computer through 


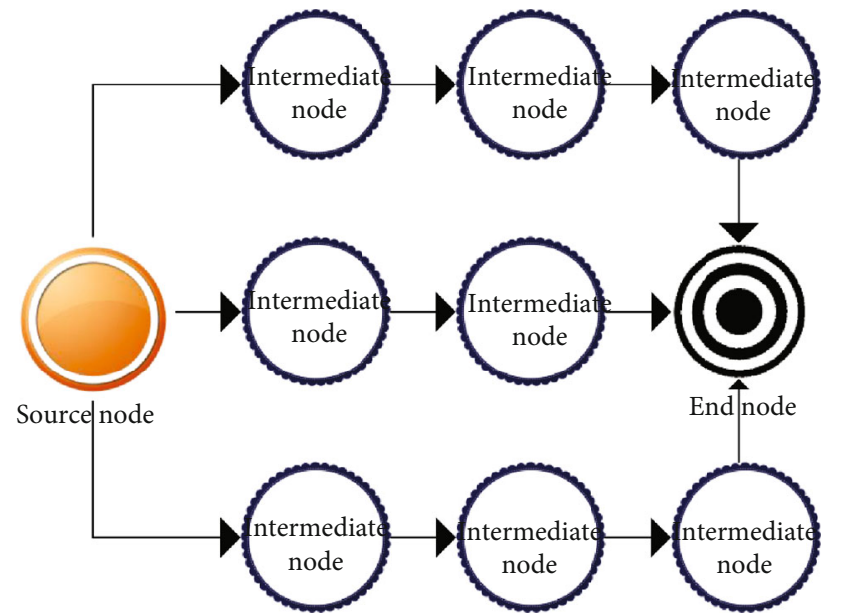

(a)

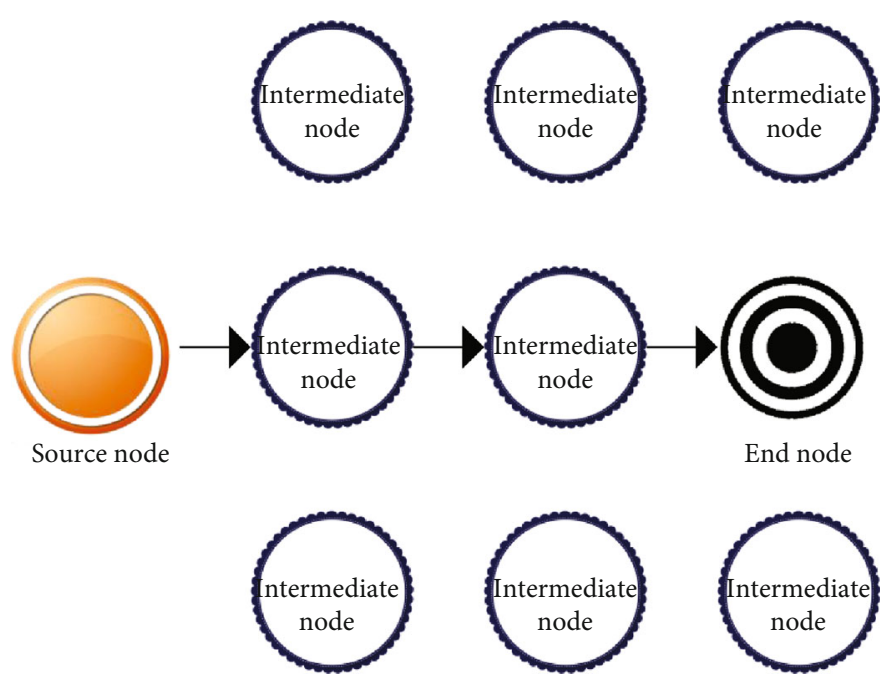

(b)
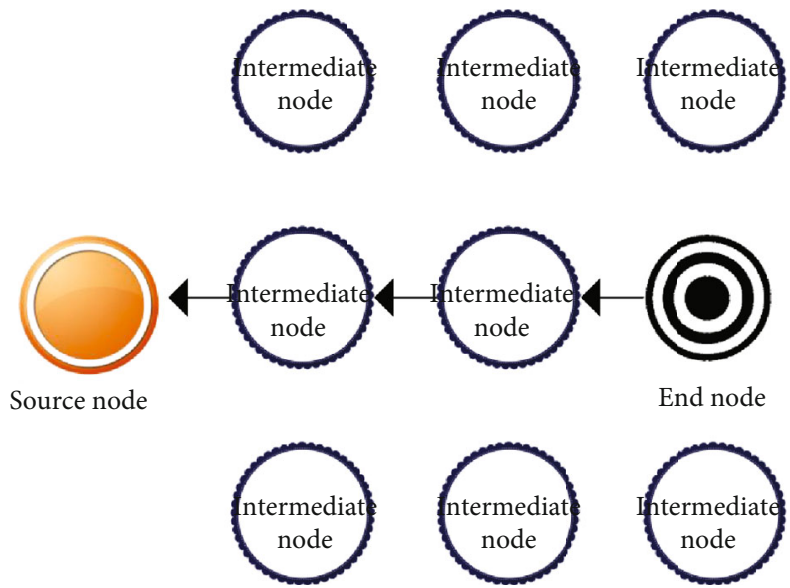

(c)

FIGURE 3: Three router discovery processes ((a) is the usual router discovery process, (b) is the router reverse data transmission process, and (c) is the router forward data transmission process).

the serial port. The whole system can record and query the situation and data on site.

The terminal node of the console has rich functions, can monitor the parameters of the current environment and the state of the equipment in real time, and can also set the parameters of the control system. Figure 5 is the hardware of the console. Among them, Advanced RISC Machines 7 (ARM7) processor is a high-speed flash memory [20]. Real-Time Clock (RTC) is a program that can set time [21]. It can run 32-bit system at the maximum clock rate. Joint Test Action Group (JTAG) [22] is an interface for testing chips and internal nodes.

In the establishment of communication network, in the wireless sensor, the responsibility of each node is to collect, calculate, and communicate the data in the system. Judging from the category of sensing equipment, sensors can be divided into two categories. One is a full function device (FFD). FFD has strong data processing capabilities and huge storage space for routing information. The other is reduce function device (RFD) [23]. The energy consumption of RFD is low, and the storage space is correspondingly small.
Using a full function device as a system gateway is the most important and common role of RFD. Simplified functional devices are used as sensor nodes to transmit data in mobile phones and transmit them to nodes in the same network layer. The two devices, RFD and FFD, work together. When the network coverage needs to be expanded, incorporating RFD equipment into the FFD can significantly reduce the energy consumption of the entire system.

The wireless sensor network routing monitoring centre (computer) and many terminal devices constitute [24], and it is necessary to use a computer to automatically control multiple devices at the same time. Therefore, the star structure network is used, and the structure diagram is shown in Figure 6 . The monitoring centre includes a computer and a master node. The master node is a full-featured service device with ZigBee, whose main function is to aggregate, transmit, and communicate data from multiple child nodes. The connected device node can use RFD or FFD. The most basic ZigBee node is the RFD node. The energy consumption of device nodes using ZigBee wireless communication technology is very low, and one AA size battery can supply 


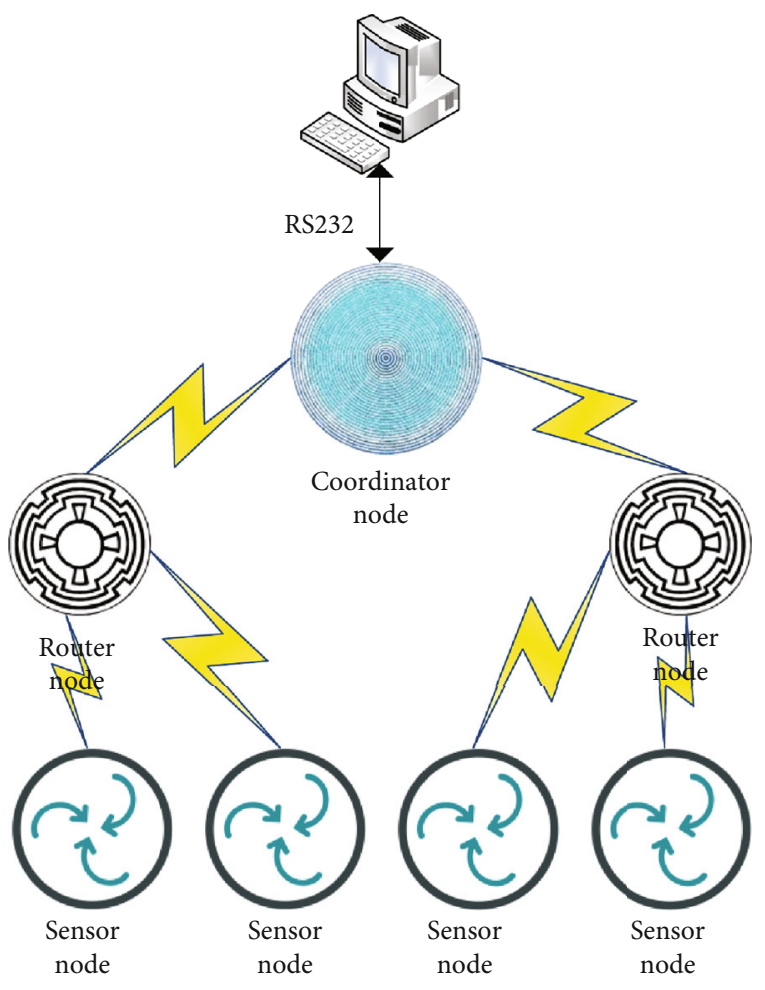

FIgURE 4: Overall structure of the ZigBee wireless sensor.

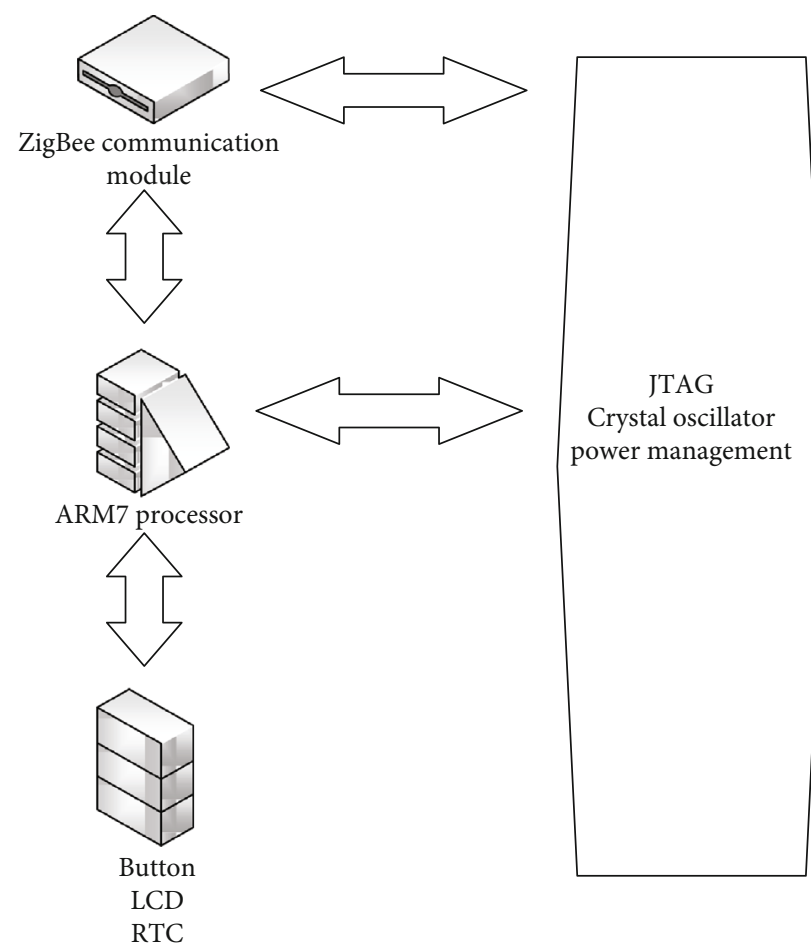

FIgURE 5: ZigBee console hardware structure diagram.

energy for a period. Independent ZigBee communication equipment generally has an effective transmission distance of less than 75 meters. To expand the communication coverage, multiple nodes can be used together.

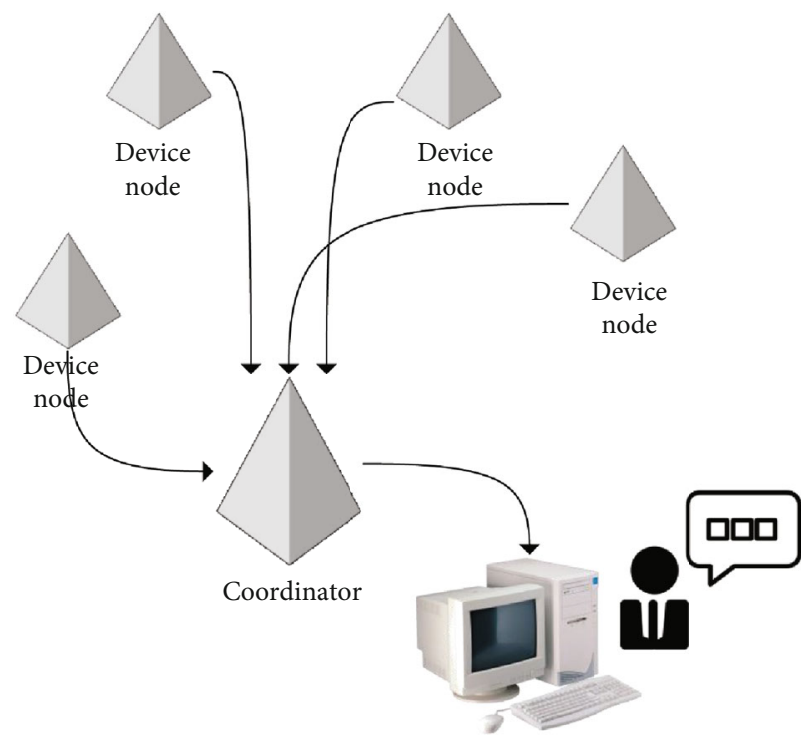

FIgURE 6: Structure diagram of communication network built with star structure.

In the design of equipment nodes, the support platform for wireless sensors is built by device nodes. This kind of device node is essentially a miniature embedded system, which includes a microcontroller unit (MCU) [25] and MCU modules of external devices, and data collection components. The structure of the power supply and communication components is shown in Figure 7. The responsibility of the data collection module [26] is to collect data within the specified monitoring range and can collect different data according to actual application areas, such as temperature, light intensity, and precipitation. The MCU is responsible for the routing protocol of the control node. Its main job is to reach the requirements of the agreement and ensure the stability of the network. The power module is powered by two $1.5 \mathrm{~V}$ alkaline batteries, which can be replaced with a smaller silver cell battery according to the actual situation. The reserved communication interface is used, and the data collection unit is set on the end node, and it is set independently. The scalability of the equipment can be improved, and the difficulty of debugging the equipment can be reduced.

In the software structure of sensor nodes, the software structure can be divided into three layers according to the use, operating system, system service, and application management. As shown in Figure 8, the access entry of the components and hardware implementing the task is controlled by the operating system. Through the control of the operating system, the functions of storage management, hardware start-up, stop processing, and task adjustment can be realized. System services typically include service components and network protocol components that provide services to corresponding applications. The nature of the system service section can be seen as an intermediate layer. From the overall analysis, node software architecture design must include communication protocols between nodes, data collection and processing of data content. 


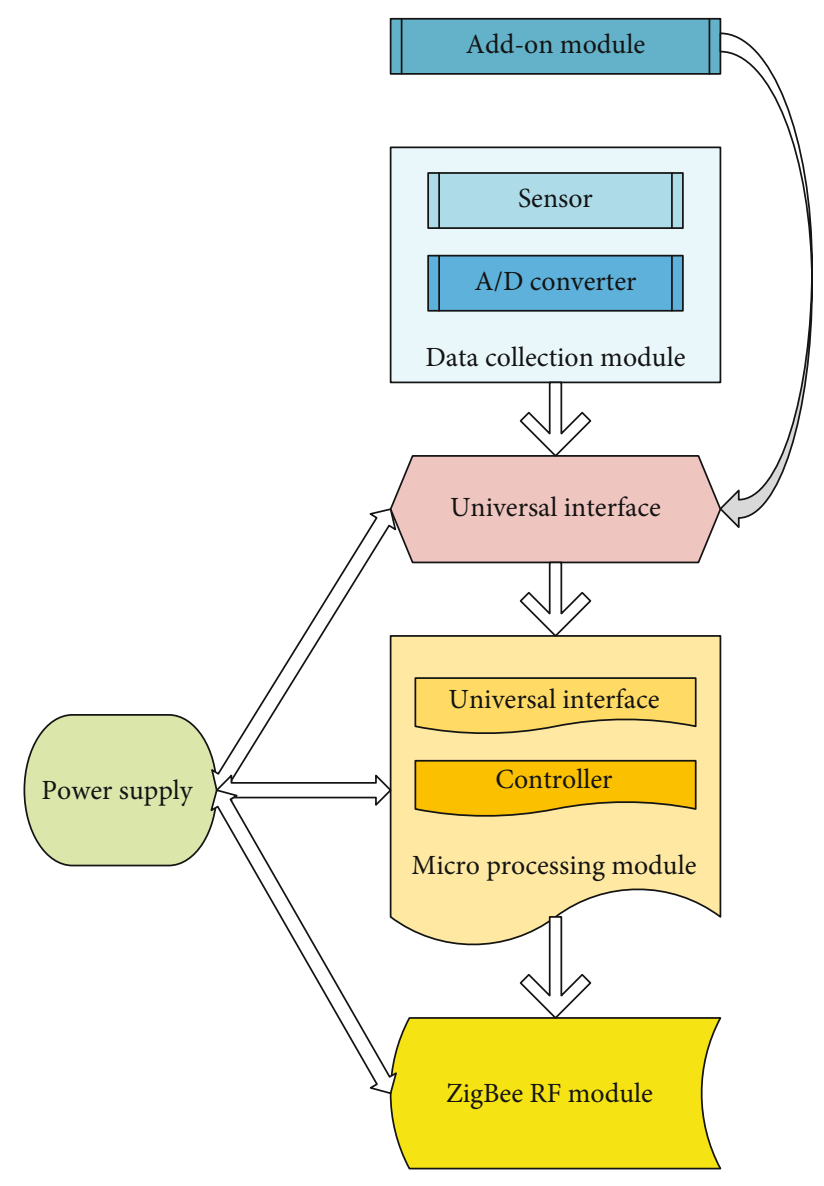

FIgURe 7: ZigBee sensor node composition diagram.

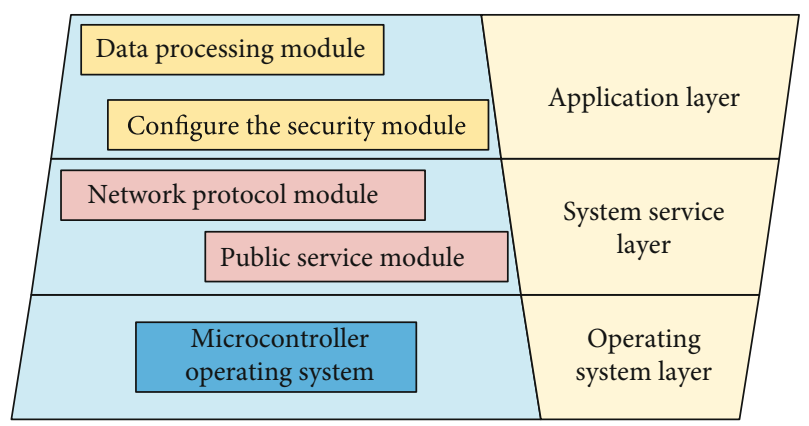

Figure 8: ZigBee wireless sensor node software composition diagram.

In the structure of communication protocol, from a specific direction, the network communication protocol can be seen as a two-dimensional architecture. In Figure 9, the communication protocol structure includes the application layer, transmission layer, network layer, data connection layer, and physical layer [27].

The physical layer is responsible for providing physical channels for data transmission. In addition, the physical layer can also assist other systems to complete the channel frequency setting and energy monitoring. Usually, the physical layer's job is to turn on or off the wireless receiver. The data link is responsible for data frame monitoring, media contact and error control, multipath multiplexing of data flow, and building one-to-one or one-to-many stable link paths. Among them, the media access control is the most important function of the connection layer. The radiofrequency $(\mathrm{RF})$ module of the node is controlled by the media access protocol, which is responsible for solving the resource allocation problem of wireless communication between sensors. Since the nodes will move, conflicts may occur between multiple nodes, and the interference of the environment will improve the error rate of the network channel of the wireless sensor. The main monitoring data of the sensor are related to energy. Therefore, the data connection layer must design a media access control method to improve the effectiveness of energy monitoring.

In the network layer control routing generation, ideally, wireless sensor networks should have on attribute address recognition function. Moreover, the network layer should provide a way to connect with external networks (such as the Internet), control system networks, etc.

In data transmission and input, Figure 10 is the data transmission process. Firstly, the transmitted data should be stored in the first in and first out cache. When processing packets with uncertain scale, the length bytes appear in the first byte. If the data size is fixed, the first byte of the first in and out cache should be the endpoint node. When valid data is stored in the first in and first out cache, synchronous bytes are sent, followed by valid loads.

Figure 11 shows the data output process. Inverse to the data transmission process, the wireless signal receives and transmits data from the line and then is processed by the hardware to achieve the exchange of data and signals. Then, data is stored in the data memory area.

When the device is in the data receiving state, the preamble and synchronization bytes in the data will be detected by the mediator and the receiving device. After matching the data, the mediator will receive the payload and performs data analysis. If the length of the data is fixed, the receiver will receive the data with reference to the default length byte. If the length of this part of the data is variable, the receiver will first save the length of the first byte record and then receive the data according to the same length.

After the match is successful, the first payload is accepted by the mediator. If the data length at this time is fixed, only the byte data of the default length will be accepted. If the data is in variable length mode at this time, the data that matches the length scale of the data will be saved.

2.3. Wireless Sensor Performance Research and Testing. The performance of the designed and built wireless transmitter needs to be verified. Two aspects can best reflect the performance of transmission technology, one is stability, and the other is transmission efficiency. The stability of the ZigBee wireless transmitter will be reflected by the real-time temperature during operation. If the temperature is too high, it means that the system is prone to be in an unstable state, and there is a possibility of failure at any time. We check the temperature through the node temperature monitoring data collected by the ZigBee wireless sensor network and compare it with the temperature monitoring data of the 


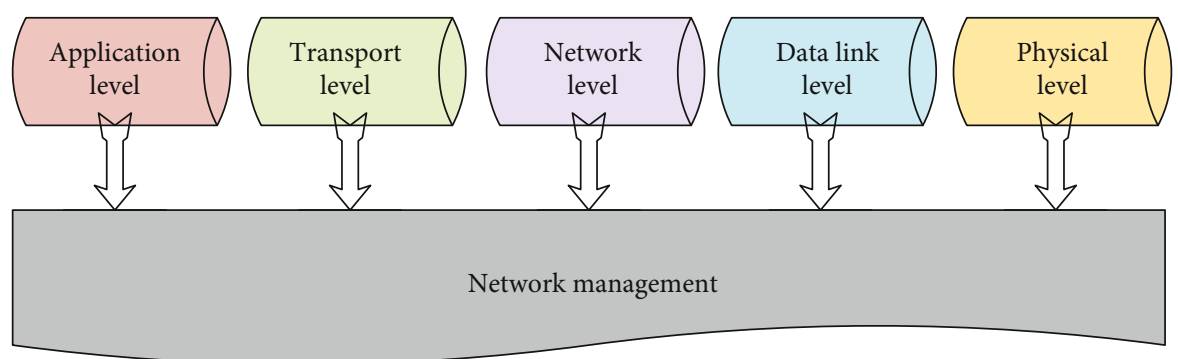

Figure 9: Architecture of ZigBee wireless sensor communication protocol.

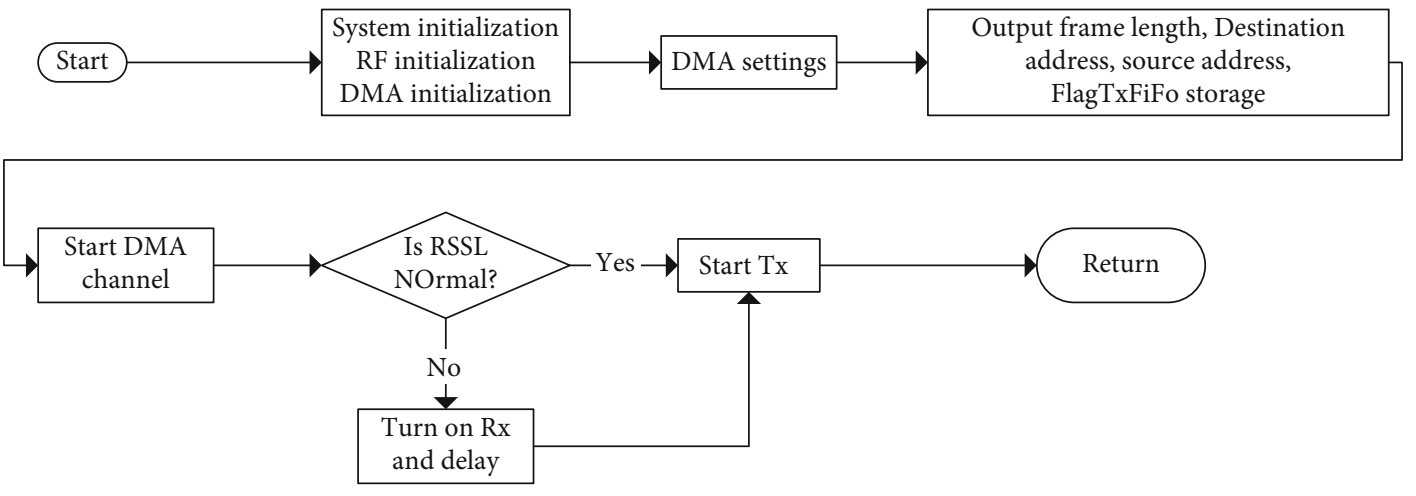

Figure 10: ZigBee wireless sensor data transmission process.

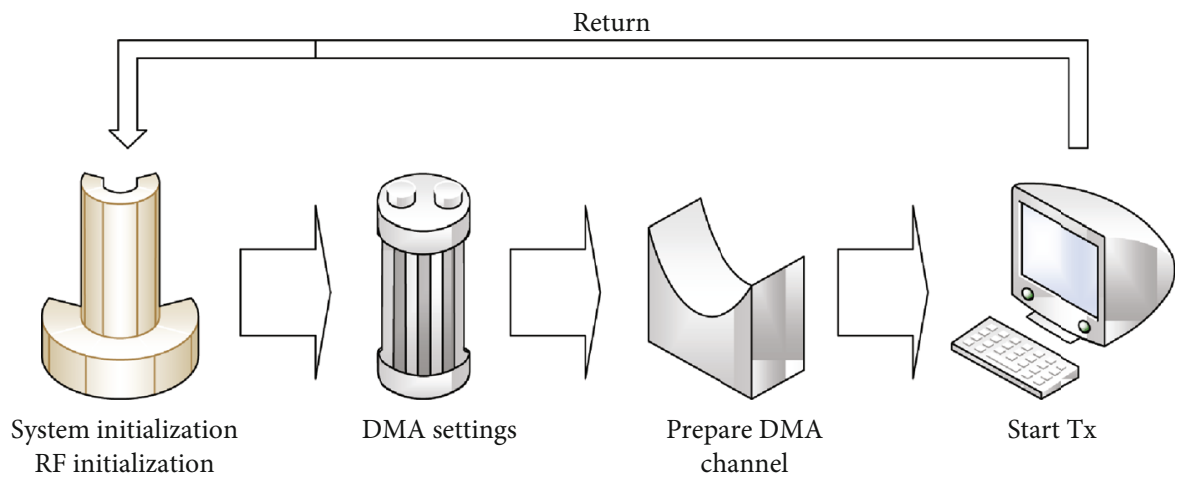

FIGURE 11: Data output process of ZigBee wireless sensor.

wired network sensor to analyze the stable performance of the ZigBee wireless sensor. Under the working conditions of high load, each component is in the state of full power operation. The machine needs high voltage to ensure operation. Long-time high-voltage operation will lead to system temperature rise. At high temperature, the mechanical properties of metal decrease rapidly. This will shorten the service life of each component, such as central processing chip, image processor, and computer graphics card. Persistent high temperatures can also lead to increased chemical performance in some specific systems, resulting in machine paralysis. In the circuit, too high temperature will also trigger off protection, resulting in the entire system to stop working, resulting in loss of production facilities.
Interfere with the transmission path by inserting obstacles in the middle of the data output device and the data receiving device. Then, the effective distance of data transmission is calculated. In the process of data transmission, whether the data is correct and whether the real-time performance can be guaranteed will determine whether the system can be used for actual automatic control. Whether the equipment parameters of the experimental site can be collected and transported correctly is a key point that must be pay attention to. Data accuracy is generally reflected in two aspects. One is whether the data collection of the sensor node is the same as the actual data or the error is small, and whether the accuracy can meet the accuracy requirements of industrial production; the other is whether a packet 
loss phenomenon occurs during wireless transmission of data.

\section{Results}

3.1. ZigBee Wireless Sensor and Wired Sensor Temperature Comparison Test. Comparing the temperature monitored by the two sensors, the data statistics of the two groups of sensors are shown in Figure 12.

By analyzing the curve data, the temperature of the traditional wired control system is higher than that of the ZigBee control system when it works. Only in the second experiment, the ZigBee temperature is slightly higher than the wired control system. The maximum temperature of the wired control system is $35.9^{\circ} \mathrm{C}$, and the minimum temperature is $35.3^{\circ} \mathrm{C}$. The maximum working temperature of ZigBee control system is $35.8^{\circ} \mathrm{C}$, and the minimum temperature is $35.2^{\circ} \mathrm{C}$. The low working temperature shows that the system has high stability and will not be powered off due to overheating. Therefore, it indicates that ZigBee control system has better stability than traditional wired control system. In the whole working process, ZigBee can maintain the temperature range of the components that is not too hot and cannot be moved due to too low temperature. So, it can better operate the whole system. ZigBee allows the system to operate at full power without breaking due to overheating.

3.2. ZigBee Wireless Sensor Data Transmission Efficiency and Distance Test. After obtaining the result that the stability of the ZigBee wireless sensor is higher than that of the wired sensor, it is necessary to test the data transmission efficiency of the ZigBee wireless sensor. In the actual application process, check whether there will be data packet loss. The research conducted experiments by increasing the distance of data transmission by setting obstacles in the data receiving, transmitting equipment, and appropriately extending the distance between the equipment. The results are shown in Figure 13.

Figure 13 shows that when there is no obstacle between the outdoor data receiving device and the transmitting device, the effective transmission efficiency of the ZigBee wireless sensor starts to drop from 40 meters. Therefore, the effective transmission distance of the ZigBee wireless sensor is about 40 meters to 50 meters when there are no obstacles.

When there are obstacles in the room that block the straight-line transmission of data, for example, when there are two brick walls blocking, the effective transmission distance of the ZigBee wireless sensor should be less than 15 meters as much as possible. When the transmission distance is farther than 20 meters, the transmission efficiency of the equipment drops greatly. When the transmission distance exceeds 40 meters, the transmission efficiency cannot be detected, so it is no longer suitable for data transmission.

Finally, when replacing the brick wall with a reinforced concrete wall, the effective transmission distance of the ZigBee wireless sensor does not exceed 10 meters. When the transmission distance exceeds 10 meters, the transmission

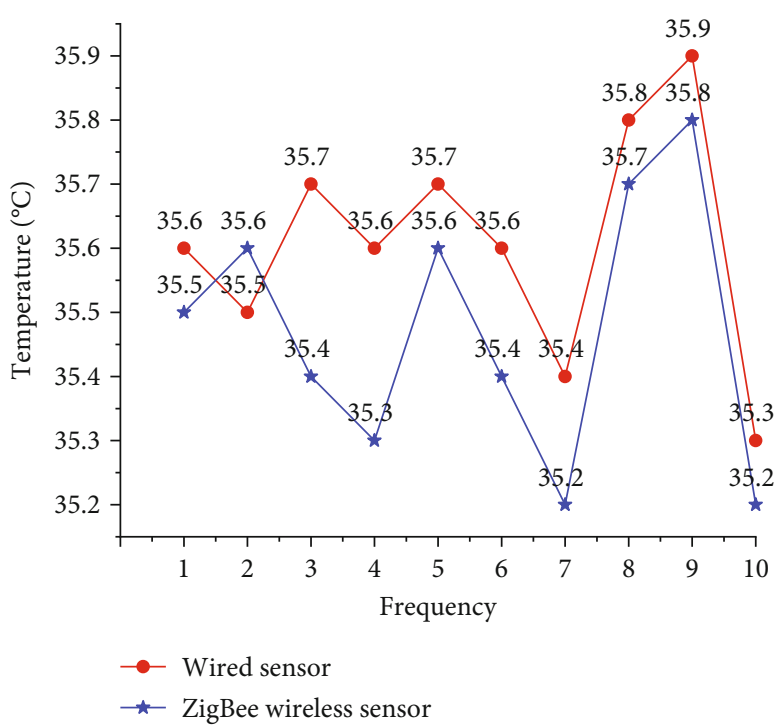

Figure 12: Comparison results of ZigBee wireless sensor and wired sensor operating temperature.

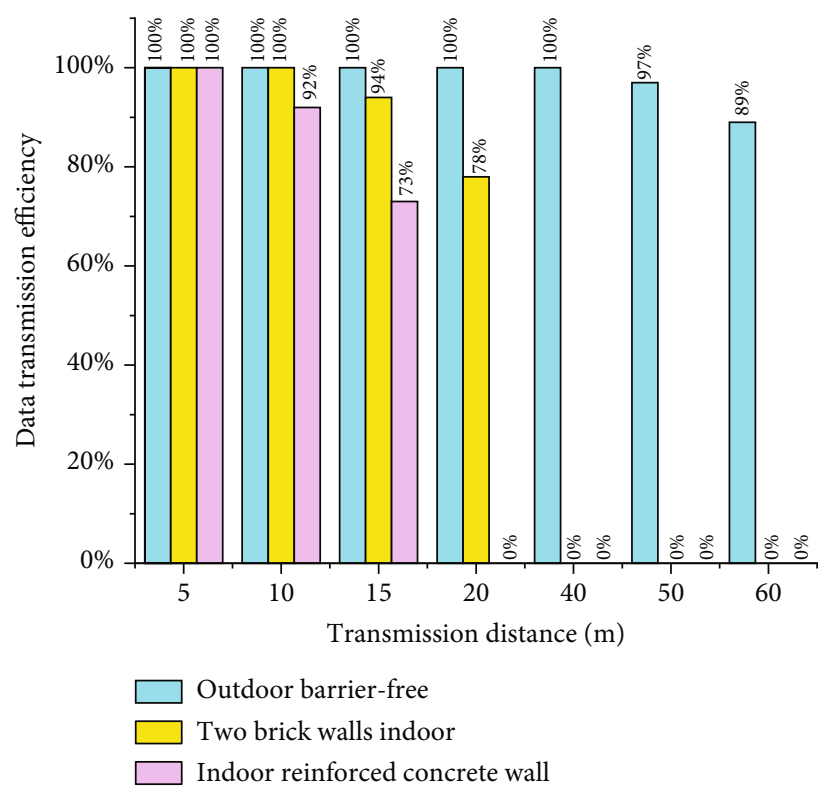

FIgURE 13: ZigBee wireless sensor data transmission efficiency test results.

efficiency drops significantly. When the transmission distance exceeds 20 meters, the transmission efficiency can no longer be detected.

Experiments show that compared with wired control, the ZigBee wireless control system is limited by transmission distance and will be blocked by obstacles between the receiver and the transmitter. But ZigBee has the advantage of fast deployment. The wired connection will occupy a large area of land because of the connection line, resulting in the disorder of the engineering site and the damage of the connection line. Wireless connection can effectively avoid these problems. 
Thus, ZigBee wireless control cannot be applied in remote automatic control. However, wireless control has better controllability than wired control. Compared with wired control, wireless control is less constrained by terrain. When operating in some areas with complex environments, cable-controlled line deployment is a problem that must be overcome, and wireless transmission can well overcome this difficulty.

\section{Conclusions}

China is a country dominated by manufacturing. In the process of gradual industrial modernization, if China wants to quickly shorten the gap in industrial strength with developed countries, it needs to improve industrial manufacturing technology and automation control technology. ZigBee wireless communication technology is used as technical support and in the establishment of wireless sensors. Necessary components such as nodes and equipment terminals in the sensor network are designed and analyzed. Subsequently, the designed ZigBee wireless sensor is used to test the automated control of a pharmaceutical factory's production workshop. The real-time temperature monitoring during the working process of the equipment and the effective data transmission distance of the equipment are studied further. In the temperature comparison between the wireless sensor and the wired sensor, it is concluded that the stability of the ZigBee wireless sensor is better than that of the wired sensor. In the transmission efficiency test, the study is conducted under the conditions of outdoor barrier-free, indoor two brick walls, and indoor reinforced concrete walls, and the maximum effective data transmission distance of three wireless sensors is obtained. Combining the two test results, wireless sensors using ZigBee wireless communication technology can be used in industrial automation control systems. At present, whether wireless sensors can be applied to industrial automation control is regarded as a research focus and measures to improve the transmission efficiency of wireless sensors have not been studied. For some large-scale industrial tasks, it is difficult to use wired transmission when the transmission distance is long. Transmission lines not only waste a lot of resources but also cause safety hazards. In this case, long-distance wireless data transmission is required. Therefore, how to improve the effective transmission distance of ZigBee wireless sensors will be the focus of subsequent research. Moreover, in the second part of the results, the environmental factors affecting wireless transmission are studied. How to solve the impact of wireless transmission environment has not been discussed. Therefore, in the future, improving the timeliness of ZigbBee wireless control system will be discussed.

\section{Data Availability}

The data used to support the findings of this study are included within the article.

\section{Conflicts of Interest}

The authors declare no conflict of interest.

\section{References}

[1] H. Haraguchi, B. Martorano, and M. Sanfilippo, "What factors drive successful industrialization? Evidence and implications for developing countries," Structural Change and Economic Dynamics, vol. 49, pp. 266-276, 2019.

[2] U. Hacioglu and G. Sevgilioglu, "The evolving role of automated systems and its cyber-security issue for global business operations in industry 4.0," International Journal of Business Ecosystem \& Strategy (2687-2293), vol. 1, no. 1, pp. 1-11, 2019.

[3] B. Zhao, "The design and research of electric automation control system based on PLC," Journal of Frontiers of Society, Science and Technology, vol. 1, no. 6, pp. 169-176, 2021.

[4] V. Aparna, "Application of DCS for level control in nonlinear system using optimization and robust algorithms," ADCAIJ: Advances in Distributed Computing and Artificial Intelligence Journal, vol. 9, no. 1, pp. 29-50, 2020.

[5] Z. Fernandez, Ó. Seijo, M. Mendicute, and I. Val, "Analysis and evaluation of a wired/wireless hybrid architecture for distributed control systems with mobility requirements," IEEE Access, vol. 7, pp. 95915-95931, 2019.

[6] S. Fu, J. Wu, H. Wen, Y. Cai, and B. Wu, "Software defined wireline-wireless cross-networks: framework, challenges, and prospects," IEEE Communications Magazine, vol. 56, no. 8, pp. 145-151, 2018.

[7] G. Debita, P. Falkowski-Gilski, M. Habrych et al., "Subjective and objective quality evaluation study of BPL-PLC wired medium," Elektronika ir Elektrotechnika, vol. 26, no. 3, pp. 13-19, 2020.

[8] S. Shrestha and S. Shakya, "Technical analysis of ZigBee wireless communication," Journal of trends in Computer Science and Smart technology (TCSST), vol. 2, no. 4, pp. 197-203, 2020.

[9] M. Abdulkarem, K. Samsudin, F. Z. Rokhani, and M. F. A Rasid, "Wireless sensor network for structural health monitoring: a contemporary review of technologies, challenges, and future direction," Structural Health Monitoring, vol. 19, no. 3, pp. 693-735, 2020.

[10] Q. Li and N. Liu, "Monitoring area coverage optimization algorithm based on nodes perceptual mathematical model in wireless sensor networks," Computer Communications, vol. 155, pp. 227-234, 2020.

[11] D. Marrero, A. Suárez, E. Macías, and V. Mena, "Extending the battery life of the ZigBee routers and coordinator by modifying their mode of operation," Sensors, vol. 20, no. 1, p. 30, 2020.

[12] S. G. Varghese, C. P. Kurian, V. I. George, A. John, V. Nayak, and A. Upadhyay, "Comparative study of ZigBee topologies for IoT-based lighting automation," IET Wireless Sensor Systems, vol. 9, no. 4, pp. 201-207, 2019.

[13] J. Xiao and J. T. Li, "Design and implementation of intelligent temperature and humidity monitoring system based on ZigBee and WiFi," Procedia Computer Science, vol. 166, pp. 419-422, 2020.

[14] V. M. Mishra and A. Kumar, "Zigbee internode communication and FPGA synthesis using mesh, star and cluster tree topological chip," in Wireless Personal Communications, pp. 1-19, Springer, 2021.

[15] Y. R. Hamdy and A. I. Alghannam, "Evaluation of ZigBee topology effect on throughput and end to end delay due to different transmission bands for IoT applications," Journal of Communications Software and Systems, vol. 16, no. 3, pp. 254-259, 2020. 
[16] Y. Yu, B. Xue, Z. Chen, and Q. Zv, "Cluster tree topology construction method based on PSO algorithm to prolong the lifetime of ZigBee wireless sensor networks," EURASIP Journal on Wireless Communications and Networking, vol. 2019, no. 1, 2019.

[17] C. E. Acosta, D. Cali, and C. Espinosa, "Autoconfiguration with global addresses using IEEE 802.15.4 standard in multihop networks," Enfoque UTE, vol. 12, no. 1, pp. 44-58, 2021.

[18] H. Y. Chang, "A connectivity-increasing mechanism of ZigBee-based IoT devices for wireless multimedia sensor networks," Multimedia Tools and Applications, vol. 78, no. 5, pp. 5137-5154, 2019.

[19] S. Meka and B. Fonseca, "Improving route selections in ZigBee wireless sensor networks," Sensors, vol. 20, no. 1, p. 164, 2020.

[20] L. Yan and S. Jianwei, "Monitoring and fault diagnosis system of wind-solar hybrid power station based on ZigBee and BP neural network," Australian Journal of Mechanical Engineering, vol. 16, supplement 1, pp. 54-60, 2018.

[21] K. Hussein, H. J. Hadi, M. R. Abdul-Mutaleb, and Y. S. Mezaal, "Low cost smart weather station using Arduino and ZigBee," Telkomnika, vol. 18, no. 1, pp. 282-288, 2020.

[22] J. M. Gómez, J. C. Mondéjar, J. R. Gómez, and J. M. Martínez, "Developing an IoT forensic methodology. A concept proposal," Forensic Science International: Digital Investigation, vol. 36, article 301114, 2021.

[23] C. H. Ke, S. Y. Hsieh, T. C. Lin, and T. H. Ho, "Efficiency network construction of advanced metering infrastructure using ZigBee," IEEE Transactions on Mobile Computing, vol. 18, no. 4, pp. 801-813, 2019.

[24] K. Xia, J. Ni, Y. Ye, P. Xu, and Y. Wang, "A real-time monitoring system using ZigBee and $4 \mathrm{G}$ communications for photovoltaic generation," CSEE Journal of Power and Energy Systems, vol. 6, no. 1, pp. 52-63, 2020.

[25] A. R. Kasar and S. Tiwari, "ZigBee on wireless sensor network," International Journal of Scientific Research and Engineering Trends, vol. 4, pp. 1327-1332, 2018.

[26] V. Saranya, S. Shankar, and G. R. Kanagachidambaresan, "Energy efficient data collection algorithm for mobile wireless sensor network," Wireless Personal Communications, vol. 105, no. 1, pp. 219-232, 2019.

[27] H. Xie, B. Yang, Z. Ren, K. Mu, X. Zhao, and B. Li, “The information security transmission method for intelligent examination based on ZigBee communication," International Journal of Information and Communication Technology, vol. 19, no. 3, pp. 258-274, 2021. 